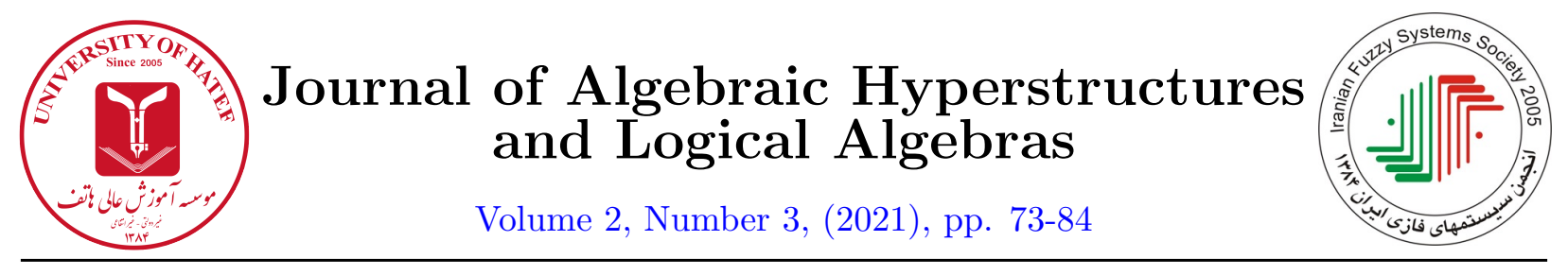

\title{
Nearness $d$-algebras
}

\author{
M.A. Öztürk ${ }^{1}$ \\ ${ }^{1}$ Department of Mathematics, Faculty of Arts and Sciences, Adryaman University, 02040 Adryaman, \\ Turkey \\ mehaliozturk@gmail.com
}

"This paper is dedicated to Professor Young Bae Jun on the occasion of his 70th birthday."

\begin{abstract}
In this paper, we have studied the notion of nearness $d$-algebras which is a generalization of nearness $B C K$ algebras (or $N B C K$-algebra for short). Therefore, it is defined that the notions of nearness $d$-subalgebra, nearness $d$-ideal in nearness $d$-algebras. Afterward, we investigated relations among them and gave some examples.
\end{abstract}

\section{Article Information}

Corresponding Author:

M.A. Öztürk;

Received: May 2021;

Accepted: June 2021;

Paper type: Original.

\section{Keywords:}

Rough sets, near sets, nearness $B C K$-algebras, nearness $d$-algebras, nearness $d$-ideals.

\section{Introduction}

Set theory is very important tool especially for engineers and mathematicians. They use set theory as a base in their studies. Researchers defined new approaches when ordinary set theory is insufficent. Because, the real world is inherently uncertain, imprecise and vague. Various problems in system identification involve characteristics which are essentially non-probabilistic in nature [27]. In response to this situation Zadeh [28] introduced fuzzy set theory as an alternative to probability theory. Then, rough set theory, proposed by Pawlak in 1982, focusesed on the uncertainty caused by indiscernible elements with different values in decision attributes [20]]. Worldwide, there has been a rapid growth in interest in rough set theory and its applications in recent years. In 1999, Molodtsov [7] suggested that one reason for these difficulties may be due to the inadequacy of the parametrization tool of the theory. Moreover, to overcome these difficulties, Molodtsov [ 7 ]

https://doi.org/10.52547/HATEF.JAHLA.2.3.6 
introduced the concept of soft set as a new mathematical tool for dealing with uncertainties that is free from the difficulties that have troubled the usual theoretical approaches.

In 2002, Peters introduced near set theory as a generalization of rough set theory. In this theory, Peters depends on the features of objects to define the nearness of objects and consequently, the classification of our universal set with respect to the available information of the objects. The concept of near set theory was motivated by image analysis and inspired by a study of the perception of the nearness of familiar physical objects. Near set theory begins with the selection of probe functions that provide a basis for describing and discerning affinities between objects in distinct perceptual granules. A probe function is a real valued function representing a feature of physical objects such as images or behaviors of individual biological organisms. In [2:3], an indiscernibility relation that depends on the features of the objects in order to define the nearness of the objects was gived. In more recent studies, it has been accepted as a generalized approach

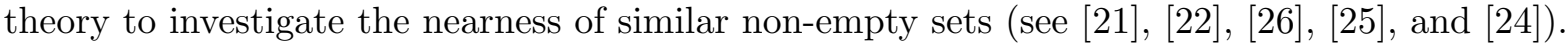

We may be used near set theory to turn elements in algebraic structures into concrete elements. Because, in the concept of ordinary algebraic structures, such a structure that consists of a nonempty set of abstract points. But this is not useful for real life problems. All researchers who study algebraic structures consider abstract elements. But using them in our study some time is insufficent. We use perceptual objects (non-abstract points) in near set theory. Perceptual objects have some features such as colour, degree of maturation for an apple. In the algebraic structures constructed on nearness approximation spaces or weak nearness approximation spaces, the basic tool is consideration of upper approximations of the subsets of perceptual objects. There are two important differences between ordinary algebraic structures and nearness algebraic structures. The first one is working with non-abstract points while the second one is considering of upper approximations of the subsets of perceptual objects for the closeness of binary operations.

The number sets of $\mathbb{N}, \mathbb{Z}, \mathbb{Q}, \mathbb{R}$, and $\mathbb{C}$ are very useful in the field of engineering. But, we emphasize that the elements of the sets of $\mathbb{N}, \mathbb{Z}, \mathbb{Q}, \mathbb{R}$ and $\mathbb{C}$ have one and only one property. Having just one feature is not valuable to study for us. Because, many things has multiple features in real life. So, we must be take attention perceptible elements which has more than one property. Since the elements of the sets of $\mathbb{N}, \mathbb{Z}, \mathbb{Q}, \mathbb{R}$ and $\mathbb{C}$ has one and only one property, upper and lower approximation's and itself of these sets are equal to each other for $r=n\left(n \in \mathbb{Z}^{+}\right)$. The sets of $\mathbb{N}, \mathbb{Z}, \mathbb{Q}, \mathbb{R}$ and $\mathbb{C}$ are algebraic structures and moreover nearness algebraic structures.

Many researchers defined algebraic structures in different sets such as fuzzy set, rough set and soft set. For example, they defined fuzzy over a group as a fuzzy group. But, nearness algebraic structures defined on a set unlike them. Therefore, we think that the nearness algebraic structure must be studied in which has property of $G \subset N_{r}(B)^{*} G$, where $G$ is a nearness algebraic structure.

In 2012, İnan and Öztürk analyzed the concept of nearness groups and investigated their basic properties [ $[2,3]$. Then, in [4], the nearness semigroups and nearness rings were established and their basic properties were investigated ( and other algebraic approaches of near sets in [III], [II], [II3] [14], [16]], [18]).

In 2015, Öztürk, Çelik Siner, and Jun introduced $B C K$-algebras on nearness approximation spaces [12]. Furthermore, the some properties of nearness $B C K$-algebras was checked out, and several examples were given about nearness $B C K$-algebras. Afterwards, in [15], Öztürk and Jun have defined quotient $N B C K$-algebras by considering an operation on the set of all cosets defined via ideals and also analyzed some properties of the quotient $N B C K$-algebras.

In this article, it is defined that the notion of nearness $d$-algebras which is a generalization of nearness $B C K$-algebras and given several examples. Furthermore, we show that the notions of nearness $d$-subalgebra, nearness $d$-ideal in nearness $d$-algebras, and investigate relations among 
them.

\section{Preliminaries}

An object description is determined by means of a tuple of function values $\Phi(x)$ associated with an object $x \in X$, which is a subset of an object space $\mathcal{O}$. Assume that $B \subseteq \mathcal{F}$ is a given set of functions representing features of sample objects $X \subseteq \mathcal{O}$. Let $\varphi_{i} \in B$, where $\varphi_{i}: \mathcal{O} \rightarrow \mathbb{R}$ (set of reals). In combination, the functions representing object features provide a basis for an object description $\Phi: \mathcal{O} \rightarrow \mathbb{R}^{L}, \Phi(x)=\left(\varphi_{1}(x), \varphi_{2}(x), \ldots, \varphi_{L}(x)\right)$ a vector containing measurements (returned values) associated with each functional value $\varphi_{i}(x)$, where the description length $|\Phi|=L$ (see [2T]).

The important thing to notice is the choice of functions $\varphi_{i} \in B$ used to describe an object of interest. Sample objects $X \subseteq \mathcal{O}$ are near each if and only if the objects have similar descriptions. Recall that each $\varphi$ defines a description of an object. Then, $\triangle_{\varphi_{i}}$ denote $\triangle_{\varphi_{i}}=\left|\varphi_{i}\left(x^{\prime}\right)-\varphi_{i}(x)\right|$, where $x^{\prime}, x \in \mathcal{O}$. The difference $\varphi$ leads to a description of the indiscernibility relation " $\sim_{B}$ " introduced by Peters in [2I] .

Definition 2.1. [2]] Let $x, x^{\prime} \in \mathcal{O}, B \subseteq \mathcal{F}$.

$$
\sim_{B}=\left\{\left(x, x^{\prime}\right) \in \mathcal{O} \times \mathcal{O} \mid \triangle_{\varphi_{i}}=0 \text { for all } \varphi_{i} \in B\right\}
$$

is called the indiscernibility relation on $\mathcal{O}$, where description length $i \leq|\Phi|$.

Comparing object descriptions is the basic idea in the near set approach to object recognition. Sets of object $X, X^{\prime}$ are called near each other if those sets contain the objects with at least partial matching descriptions.

Definition 2.2. [2]] Let $X, X^{\prime} \subseteq \mathcal{O}, B \subseteq \mathcal{F}$. Then $X$ is called near $X^{\prime}$ if there exist $x \in X, x^{\prime} \in$ $X^{\prime}, \varphi_{i} \in B$ such that $x \sim_{\varphi_{i}} x^{\prime}$.

A weak nearness approximation space is a tuple $\left(\mathcal{O}, \mathcal{F}, \sim_{B_{r}}, N_{r}(B)\right)$, where the approximation space is defined with respect to a set of perceived objects $\mathcal{O}$, set of probe functions $\mathcal{F}$ representing object features, $\sim_{B_{r}}$ indiscernibility relation $B_{r}$ defined relative to $B_{r} \subseteq B \subseteq \mathcal{F}$, and collection of partitions (families of neighbourhoods) $N_{r}(B)$. This relation $\sim_{B_{r}}$ defines a partition of $\mathcal{O}$ into non-empty, pairwise disjoint subsets that are equivalence classes denoted by $[x]_{B_{r}}$, where $[x]_{B_{r}}=\left\{x^{\prime} \in \mathcal{O} \mid x \sim_{B_{r}} x^{\prime}\right\}$. These classes form a new set called the quotient set $\mathcal{O} / \sim_{B_{r}}$, where $\mathcal{O} / \sim_{B_{r}}=\left\{[x]_{B_{r}} \mid x \in \mathcal{O}\right\}$. In effect, each choice of probe functions $B_{r}$ defines a partition $\xi_{\mathcal{O}, B_{r}}$ on a set of objects $\mathcal{O}$, namely, $\xi_{\mathcal{O}, B_{r}}=\mathcal{O} / \sim_{B_{r}}$. We consider $X \subseteq \mathcal{O}$, then upper approximation of $X$ defined by $N_{r}(B)^{-} X=\bigcup_{[x]_{B_{r}} \cap X \neq \varnothing}[x]_{B_{r}}$, and lower approximation of $X$ defined by $N_{r}(B)_{-} X=\bigcup_{[x]_{B_{r}} \subseteq X}[x]_{B_{r}}$, (see [I0], [2I]).

Theorem 2.3. [15] Let $\left(\mathcal{O}, \mathcal{F}, \sim_{B_{r}}, N_{r}(B)\right)$ be a weak nearness approximation space and $X, Y \subset \mathcal{O}$, then the following statements hold;

i) $N_{r}(B)_{-} X \subseteq X \subseteq N_{r}(B)^{-} X$,

ii) $N_{r}(B)^{-}(X \cup Y)=\left(N_{r}(B)^{-} X\right) \cup\left(N_{r}(B)^{-} Y\right)$,

iii) $N_{r}(B)_{-}(X \cup Y) \supseteq\left(N_{r}(B)_{-} X\right) \cup\left(N_{r}(B)_{-} Y\right)$,

iv) $N_{r}(B)^{-}(X \cap Y) \subseteq\left(N_{r}(B)^{-} X\right) \cap\left(N_{r}(B)^{-} Y\right)$,

v) $N_{r}(B)_{-}(X \cap Y)=\left(N_{r}(B)_{-} X\right) \cap\left(N_{r}(B)_{-} Y\right)$,

vi) $X \subseteq Y$ implies $N_{r}(B)^{-} X \subseteq N_{r}(B)^{-} Y$,

vii) $X \subseteq Y$ implies $N_{r}(B)_{-} X \subseteq N_{r}(B)_{-} Y$. 
Definition 2.4. [15] Let $\left(\mathcal{O}, \mathcal{F}, \sim_{B_{r}}, N_{r}(B)\right)$ be a weak nearness approximation space; $\emptyset \neq \mathcal{X} \subseteq$ $\mathcal{O}$, and 0 be a constant on $\mathcal{O}$. A subset $\mathcal{X}$ of the set $\mathcal{O}$ is called $B C K$-algebra on nearness approximation space $\mathcal{O}$ or $N B C K$-algebra for short if the following properties are satisfied for all $a, b, c \in \mathcal{X}$

$N B C I-i)((a \circledast b) \circledast(a \circledast c)) \circledast(c \circledast b)=0$ property holds in $N_{r}(B)^{-} \mathcal{X}$,

$N B C I-i i)(a \circledast(a \circledast b)) \circledast b=0$ property holds in $N_{r}(B)^{-} \mathcal{X}$,

$N B C I-i i i) a \circledast a=0$ property holds in $N_{r}(B)^{-} \mathcal{X}$,

$N B C K-i v) 0 \circledast a=0$ property holds in $N_{r}(B)^{-} \mathcal{X}$,

$N B C I-v)$ If $a \circledast b=0$ and $b \circledast a=0$, then $a=b$ property holds in $\mathcal{X}$,

where $\circledast: \mathcal{X} \times \mathcal{X} \rightarrow N_{r}(B)^{-} \mathcal{X}$ is a well-defined operation.

"§" relation defined on $\mathcal{X}$, for all $a, b \in \mathcal{X}, a \preceq b: \Leftrightarrow a \circledast b=0$.

NBCK-algebra again can be defined with this relation:

$\left.N B C I-i^{\prime}\right)(a \circledast b) *(a \circledast c) \preceq c \circledast b$ property holds in $N_{r}(B)^{-} \mathcal{X}$,

$\left.N B C I-i i^{\prime}\right) a *(a \circledast b) \preceq b$ property holds in $N_{r}(B)^{-} \mathcal{X}$,

$\left.N B C I-i i i^{\prime}\right) a \preceq a$ property holds in $N_{r}(B)^{-} \mathcal{X}$,

$\left.N B C K-i v^{\prime}\right) 0 \preceq a$ property holds in $N_{r}(B)^{-} \mathcal{X}$,

$\left.N B C I-v^{\prime}\right)$ If $a \preceq b$ and $b \preceq a$, then $a=b$ property holds in $\mathcal{X}$,

$\left.N B C I-v i^{\prime}\right) a \preceq b \Leftrightarrow a \circledast b=0$ property holds in $N_{r}(B)^{-} \mathcal{X}$.

Definition 2.5. [15] Let $\mathcal{X}$ be an $N B C K$-algebra, and $S$ be a non-empty subset of $\mathcal{X}$. Then, $S$ is called a subalgebra of $\mathcal{X}$ if $0 \in N_{r}(B)^{-} S$ and $a \circledast b \in N_{r}(B)^{-} S$ for all $a, b \in S$.

For other notions and definitions not mentioned in this paper, the readers are referred to [5] $[6],[\underline{8}],[9],[12],[16],[15]$ and [22].

\section{$3 \quad$ Nearness $d$-algebras}

Definition 3.1. Let $\left(\mathcal{O}, \mathcal{F}, \sim_{B_{r}}, N_{r}(B)\right)$ be a weak nearness approximation space; $\emptyset \neq \mathcal{X} \subseteq \mathcal{O}$, and 0 be a constant on $\mathcal{O}$. A subset $\mathcal{X}$ of the set $\mathcal{O}$ is called d-algebra on weak nearness approximation space $\mathcal{O}$ or nearness $d$-algebra for short if the following properties are satisfied for all $a, b, c \in \mathcal{X}$

$N d-i) a \circledast a=0$ property holds in $N_{r}(B)^{-} \mathcal{X}$,

$N d-i i) 0 \circledast a=0$ property holds in $N_{r}(B)^{-} \mathcal{X}$,

$N d-i i i)$ If $a \circledast b=0$ and $b \circledast a=0$, then $a=b$ property holds in $\mathcal{X}$, where $\circledast: \mathcal{X} \times \mathcal{X} \rightarrow$ $N_{r}(B)^{-} \mathcal{X}$ is a well-defined operation.

A nearness $B C K$-algebra is a nearness $d$-algebra $(\mathcal{X}, *, 0)$ satisfying the following additional axioms:

$N B C K-i)((a \circledast b) \circledast(a \circledast c)) \circledast(c \circledast b)=0$ property holds in $N_{r}(B)^{-} \mathcal{X}$,

$N B C K-i i)(a \circledast(a \circledast b)) \circledast b=0$ property holds in $N_{r}(B)^{-} \mathcal{X}$,

for all $a, b, c \in \mathcal{X}$. Therefore, nearness $d$-algebra is a generalization of nearness $B C K$-algebras.

Example 3.2. Let $\mathcal{O}=\{0, a, b, c, d, e\}$ be a set of perceptual objects, $r=1, B=\left\{\varphi_{1}, \varphi_{2}, \varphi_{3}\right\} \subseteq \mathcal{F}$ be a set of probe functions, and $\mathcal{X}=\{a, b, c\} \subset \mathcal{O}$. Values of the probe functions

$$
\begin{aligned}
& \varphi_{1}: \mathcal{O} \rightarrow V_{1}=\left\{\beta_{1}, \beta_{2}, \beta_{3}, \beta_{4}\right\}, \\
& \varphi_{2}: \mathcal{O} \rightarrow V_{2}=\left\{\beta_{2}, \beta_{3}, \beta_{4}\right\}, \\
& \varphi_{3}: \mathcal{O} \rightarrow V_{3}=\left\{\beta_{1}, \beta_{2}, \beta_{3}, \beta_{4}, \beta_{5}\right\}
\end{aligned}
$$


are given in Table 1.

\section{Table 1}

\begin{tabular}{c|cccccc} 
& 0 & $a$ & $b$ & $c$ & $d$ & $e$ \\
\hline$\varphi_{1}$ & $\beta_{1}$ & $\beta_{1}$ & $\beta_{2}$ & $\beta_{3}$ & $\beta_{1}$ & $\beta_{4}$ \\
$\varphi_{2}$ & $\beta_{2}$ & $\beta_{3}$ & $\beta_{2}$ & $\beta_{3}$ & $\beta_{3}$ & $\beta_{4}$ \\
$\varphi_{3}$ & $\beta_{2}$ & $\beta_{3}$ & $\beta_{2}$ & $\beta_{4}$ & $\beta_{1}$ & $\beta_{5}$
\end{tabular}

Let us now determine the near equivalence classes according to the indiscernibility relation $\sim_{B_{r}}$ of elements of $\mathcal{O}$ :

$$
\begin{aligned}
& {[0]_{\varphi_{1}}=\left\{y \in \mathcal{O} \mid \varphi_{1}(y)=\varphi_{1}(0)=\beta_{1}\right\}=\{0, a, d\}=[a]_{\varphi_{1}}=[d]_{\varphi_{1}},} \\
& {[b]_{\varphi_{1}}=\left\{y \in \mathcal{O} \mid \varphi_{1}(y)=\varphi_{1}(b)=\beta_{2}\right\}=\{b\}} \\
& {[c]_{\varphi_{1}}=\left\{y \in \mathcal{O} \mid \varphi_{1}(y)=\varphi_{1}(c)=\beta_{3}\right\}=\{c\}} \\
& {[e]_{\varphi_{1}}=\left\{y \in \mathcal{O} \mid \varphi_{1}(y)=\varphi_{1}(e)=\beta_{4}\right\}=\{e\} .}
\end{aligned}
$$

Then, we get $\xi_{\varphi_{1}}=\left\{[0]_{\varphi_{1}},[b]_{\varphi_{1}},[c]_{\varphi_{1}},[e]_{\varphi_{1}}\right\}$.

$$
\begin{aligned}
& {[0]_{\varphi_{2}}=\left\{y \in \mathcal{O} \mid \varphi_{2}(y)=\varphi_{2}(0)=\beta_{2}\right\}=\{0, b\}=[b]_{\varphi_{2}},} \\
& {[a]_{\varphi_{2}}=\left\{y \in \mathcal{O} \mid \varphi_{2}(y)=\varphi_{2}(a)=\beta_{3}\right\}=\{a, c, d\},=[c]_{\varphi_{2}}=[d]_{\varphi_{2}},} \\
& {[e]_{\varphi_{2}}=\left\{y \in \mathcal{O} \mid \varphi_{2}(y)=\varphi_{2}(d)=\beta_{4}\right\}=\{e\} .}
\end{aligned}
$$

Thus, we have $\xi_{\varphi_{2}}=\left\{[0]_{\varphi_{2}},[a]_{\varphi_{2}},[e]_{\varphi_{2}}\right\}$.

$$
\begin{aligned}
& {[0]_{\varphi_{3}}=\left\{y \in \mathcal{O} \mid \varphi_{3}(y)=\varphi_{3}(0)=\beta_{2}\right\}=\{0, b\}=[b]_{\varphi_{3}},} \\
& {[a]_{\varphi_{3}}=\left\{y \in \mathcal{O} \mid \varphi_{3}(y)=\varphi_{3}(a)=\beta_{3}\right\}=\{a\},} \\
& {[c]_{\varphi_{2}}=\left\{y \in \mathcal{O} \mid \varphi_{2}(y)=\varphi_{2}(c)=\beta_{4}\right\}=\{c\},} \\
& {[d]_{\varphi_{3}}=\left\{y \in \mathcal{O} \mid \varphi_{3}(y)=\varphi_{3}(d)=\beta_{1}\right\}=\{d\},} \\
& {[e]_{\varphi_{3}}=\left\{y \in \mathcal{O} \mid \varphi_{3}(y)=\varphi_{3}(e)=\beta_{5}\right\}=\{e\} .}
\end{aligned}
$$

From hence, we obtain that $\xi_{\varphi_{3}}=\left\{[0]_{\varphi_{3}},[a]_{\varphi_{3}},[c]_{\varphi_{3}}[d]_{\varphi_{3}},[e]_{\varphi_{3}}\right\}$. Therefore, for $r=1$, a set of partitions of $\mathcal{O}$ is $N_{1}(B)=\left\{\xi_{\varphi_{1}}, \xi_{\varphi_{2}}, \xi_{\varphi_{3}}\right\}$. Then, we can write

$$
N_{1}(B)^{-} \mathcal{X}=\bigcup_{[x]_{\varphi_{i}} \cap \mathcal{X} \neq \emptyset}^{[x]_{\varphi_{i}}}=\{0, a, b, c, d\} .
$$

Considering the following table of operation:

Table 2

\begin{tabular}{c|lllll}
$\circledast$ & 0 & $a$ & $b$ & $c$ & $d$ \\
\hline 0 & 0 & 0 & 0 & 0 & 0 \\
$a$ & $a$ & 0 & 0 & 0 & 0 \\
$b$ & $b$ & $b$ & 0 & $d$ & $d$ \\
$c$ & $c$ & $a$ & $a$ & 0 & 0 \\
$d$ & $d$ & $d$ & $d$ & $d$ & 0
\end{tabular}


In that case; $\mathcal{X}:=\{a, b, c\}$ is a d-algebra by Definition [3.]. But, $\mathcal{X}$ is not a nearness BCKalgebra, since $(b \circledast(b \circledast c)) \circledast c=(b \circledast d) \circledast c=d \circledast c=d \neq 0$.

Definition 3.3. Let $\mathcal{X}$ be a nearness d-algebra, and $S$ be a non-empty subset of $\mathcal{X}$. Then, $S$ is called a nearness d-subalgebra of $\mathcal{X}$ if $a \circledast b \in N_{r}(B)^{-} S$ for all $a, b \in S$. Also, $S$ is called an upper-near d-subalgebra of $\mathcal{X}$ if $0 \in N_{r}(B)^{-} S$ and $a \circledast b \in N_{r}(B)^{-} S$ for all $a, b \in N_{r}(B)^{-} S$.

Example 3.4. From Example [马, we have $N_{1}(B)^{-} \mathcal{X}=\{0, a, b, c, d\} \subset \mathcal{O}$. Then, we can write

$$
N_{1}(B)^{-}\left(N_{1}(B)^{-} \mathcal{X}\right)=\bigcup_{[x]_{\varphi_{i}} \cap N_{1}(B)^{-} \mathcal{X} \neq \emptyset}^{[x]_{\varphi_{i}}}=\{0, a, b, c, d\} .
$$

In that case; $N_{1}(B)^{-} \mathcal{X}=\{0, a, b, c, d\}$ is a nearness d-algebra by Table 2. Let $S:=\{b, c\} \subset \mathcal{X}$. Thus, we get

$$
N_{1}(B)^{-} S=\bigcup_{\left.[x]_{\varphi_{i}} \cap S \neq \emptyset\right]_{\varphi_{i}}}=\{0, b, c, d\}
$$

Then, $S:=\{b, c\}$ isn't a nearness d-subalgebra of $\mathcal{X}$, for $c \circledast b=a \notin N_{1}(B)^{-} S$ by Table 2. If $S:=\{a, c\} \subset \mathcal{X}$ then,

$$
N_{1}(B)^{-} S=\bigcup_{[x]_{\varphi_{i}} \cap S \neq \emptyset}^{[x]_{\varphi_{i}}}=\{0, a, c, d\}
$$

From Table 2, $S:=\{b, c\}$ is a nearness d-subalgebra of $\mathcal{X}$.

Definition 3.5. Let $\mathcal{X}$ be a d-algebra on $\mathcal{O}, B_{r} \subseteq \mathcal{F}$ where $r \leq|B|$ and $B \subseteq \mathcal{F}$, $\sim_{B_{r}}$ be an indiscernibility relation on $\mathcal{O}$. Then, $\sim_{B_{r}}$ is called a congruence indiscernibility relation on $\mathcal{X}$, if $x \sim_{B_{r}} y$, where $x, y \in \mathcal{X}$ implies $(x \circledast b) \sim_{B_{r}}(y \circledast b)$, and $(b \circledast x) \sim_{B_{r}}(b \circledast y)$ for all $a, b \in \mathcal{X}$.

Theorem 3.6. Let $\mathcal{X}$ be a nearness d-algebra, $\sim_{B_{r}}$ be a congruence indiscernibility relation on $\mathcal{X}$, and $S$ be a non-empty subset of $\mathcal{X}$. If $a \circledast b \in S$ for all $a, b \in S$, then $S$ is an upper-near $d$-subalgebra of $\mathcal{X}$.

Proof. Let $a, b \in N_{r}(B)^{-} S$. Then, we have $[a]_{B_{r}} \cap S \neq \emptyset$ and $[b]_{B_{r}} \cap S \neq \emptyset$. Hence, there exists elements $x, y \in S$ such that $x \in[a]_{B_{r}}$ and $y \in[b]_{B_{r}}$. In this case, $x \circledast y \in S$ by hypothesis, $a \sim_{B_{r}} x$ and $b \sim_{B_{r}} y$, thus $(a \circledast b) \sim_{B_{r}}(x \circledast b)$ and $(x \circledast b) \sim_{B_{r}}(x \circledast y)$ and so $(a \circledast b) \sim_{B_{r}}(x \circledast y)$. Hence $x \circledast y \in[a \circledast b]_{B_{r}}$, and so $[a \circledast b]_{B_{r}} \cap S \neq \emptyset$. Therefore, we get $a \circledast b \in N_{r}(B)^{-} S$. Also, $a \circledast a=0 \in N_{r}(B)^{-} S$ by Definition 3.3 .

Definition 3.7. Let $(\mathcal{X}, \circledast, 0)$ be a nearness d-algebra, and $I$ be a non-empty subset of $\mathcal{X}$. Then,

i) $A$ set $I$ is called a nearness d-ideal of $\mathcal{X}$ if it satisfies:

$N I 1) a \circledast b \in N_{r}(B)^{-} I$ and $b \in I$ implies $a \in I$ for all $a, b \in \mathcal{X}$,

NI2) $a \in I$ and $b \in \mathcal{X}$ imply $a \circledast b \in N_{r}(B)^{-} I$.

ii) A set $I$ is called an upper-near d-ideal of $\mathcal{X}$ if it satisfies:

$U N I 1) \quad 0 \in N_{r}(B)^{-} I$,

$U N I 2) a \circledast b \in N_{r}(B)^{-} I$ and $b \in I$ implies $a \in I$ for all $a, b \in \mathcal{X}$,

$U N I 3) a \in N_{r}(B)^{-} I$ and $b \in N_{r}(B)^{-} \mathcal{X}$ implies $a \circledast b \in N_{r}(B)^{-} I$. 
Example 3.8. Let $\mathcal{O}=\{0, a, b, c, d, e, f\}$ be a set of perceptual objects, $r=1, B=\left\{\varphi_{1}, \varphi_{2}, \varphi_{3}\right\} \subseteq$ $\mathcal{F}$ be a set of probe functions, and $\mathcal{X}=\{a, b, c, d, e\} \subset \mathcal{O}, I=\{a, b, c\} \subset \mathcal{X}$. Values of the probe functions

$$
\begin{aligned}
& \varphi_{1}: \mathcal{O} \rightarrow V_{1}=\left\{\beta_{1}, \beta_{2}, \beta_{3}, \beta_{4}, \beta_{5}\right\}, \\
& \varphi_{2}: \mathcal{O} \rightarrow V_{2}=\left\{\beta_{1}, \beta_{2}, \beta_{3}, \beta_{4}, \beta_{5}\right\}, \\
& \varphi_{3}: \mathcal{O} \rightarrow V_{3}=\left\{\beta_{1}, \beta_{2}, \beta_{3}, \beta_{4}\right\}
\end{aligned}
$$

are given in Table 3 .

\section{Table 3}

\begin{tabular}{c|ccccccc} 
& 0 & $a$ & $b$ & $c$ & $d$ & $e$ & $f$ \\
\hline$\varphi_{1}$ & $\beta_{1}$ & $\beta_{1}$ & $\beta_{2}$ & $\beta_{2}$ & $\beta_{3}$ & $\beta_{5}$ & $\beta_{4}$ \\
$\varphi_{2}$ & $\beta_{1}$ & $\beta_{4}$ & $\beta_{4}$ & $\beta_{2}$ & $\beta_{3}$ & $\beta_{2}$ & $\beta_{5}$ \\
$\varphi_{3}$ & $\beta_{2}$ & $\beta_{1}$ & $\beta_{4}$ & $\beta_{1}$ & $\beta_{2}$ & $\beta_{4}$ & $\beta_{3}$
\end{tabular}
of $\mathcal{O}$ :

We find the near equivalence classes according to the indiscernibility relation of $\sim_{B_{r}}$ of elements

$$
\begin{gathered}
{[0]_{\varphi_{1}}=\left\{y \in \mathcal{O} \mid \varphi_{1}(y)=\varphi_{1}(0)=\beta_{1}\right\}=\{0, a\}=[a]_{\varphi_{1}},} \\
{[b]_{\varphi_{1}}=\left\{y \in \mathcal{O} \mid \varphi_{1}(y)=\varphi_{1}(b)=\beta_{2}\right\}=\{b, c\}=[c]_{\varphi_{1}},} \\
{[d]_{\varphi_{1}}=\left\{y \in \mathcal{O} \mid \varphi_{1}(y)=\varphi_{1}(d)=\beta_{3}\right\}=\{d\}} \\
{[e]_{\varphi_{1}}=\left\{y \in \mathcal{O} \mid \varphi_{1}(y)=\varphi_{1}(e)=\beta_{5}\right\}=\{e\}} \\
{[f]_{\varphi_{1}}=\left\{y \in \mathcal{O} \mid \varphi_{1}(y)=\varphi_{1}(f)=\beta_{4}\right\}=\{f\} .}
\end{gathered}
$$

Then, we get that $\xi_{\varphi_{1}}=\left\{[0]_{\varphi_{1}},[b]_{\varphi_{1}},[d]_{\varphi_{1}},[e]_{\varphi_{1}},[f]_{\varphi_{1}}\right\}$.

$$
\begin{aligned}
& {[0]_{\varphi_{2}}=\left\{y \in \mathcal{O} \mid \varphi_{2}(y)=\varphi_{2}(0)=\beta_{1}\right\}=\{0\},} \\
& {[a]_{\varphi_{2}}=\left\{y \in \mathcal{O} \mid \varphi_{2}(y)=\varphi_{2}(a)=\beta_{4}\right\}=\{a, b\}=[b]_{\varphi_{2}},} \\
& {[c]_{\varphi_{2}}=\left\{y \in \mathcal{O} \mid \varphi_{2}(y)=\varphi_{2}(c)=\beta_{2}\right\}=\{c, e\}=[e]_{\varphi_{2}},} \\
& {[d]_{\varphi_{2}}=\left\{y \in \mathcal{O} \mid \varphi_{2}(y)=\varphi_{2}(d)=\beta_{3}\right\}=\{d\},} \\
& {[f]_{\varphi_{2}}=\left\{y \in \mathcal{O} \mid \varphi_{2}(y)=\varphi_{2}(d)=\beta_{5}\right\}=\{f\} .}
\end{aligned}
$$

Thus, we have that $\xi_{\varphi_{2}}=\left\{[0]_{\varphi_{2}},[a]_{\varphi_{2}},[c]_{\varphi_{2}},[d]_{\varphi_{2}},[f]_{\varphi_{2}}\right\}$.

$$
\begin{aligned}
& {[0]_{\varphi_{3}}=\left\{y \in \mathcal{O} \mid \varphi_{3}(y)=\varphi_{3}(0)=\beta_{2}\right\}=\{0, d\},} \\
& {[a]_{\varphi_{3}}=\left\{y \in \mathcal{O} \mid \varphi_{3}(y)=\varphi_{3}(a)=\beta_{1}\right\}=\{a, c\}=[c]_{\varphi_{3}},} \\
& {[b]_{\varphi_{3}}=\left\{y \in \mathcal{O} \mid \varphi_{3}(y)=\varphi_{3}(b)=\beta_{4}\right\}=\{b, e\}=[e]_{\varphi_{3}},} \\
& {[f]_{\varphi_{3}}=\left\{y \in \mathcal{O} \mid \varphi_{3}(y)=\varphi_{3}(f)=\beta_{3}\right\}=\{f\} .}
\end{aligned}
$$

From hence, we obtain that $\xi_{\varphi_{3}}=\left\{[0]_{\varphi_{3}},[a]_{\varphi_{3}},[b]_{\varphi_{3}},[f]_{\varphi_{3}}\right\}$. Therefore, for $r=1$, a set of partitions of $\mathcal{O}$ is $N_{1}(B)=\left\{\xi_{\varphi_{1}}, \xi_{\varphi_{2}}, \xi_{\varphi_{3}}\right\}$. Then, we can write

$$
N_{1}(B)^{-} \mathcal{X}=\bigcup_{[x]_{\varphi_{i}} \cap[x]_{\varphi_{i}} \cap \mathcal{X} \neq \emptyset}=\{0, a, b, c, d, e, f\} .
$$


Furthermore,

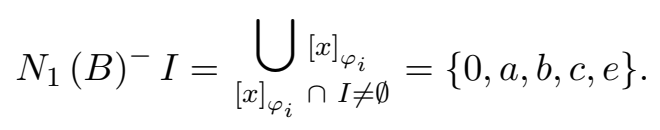

Considering the following table of operation:

Table 4

\begin{tabular}{c|lllllll}
$\circledast$ & 0 & $a$ & $b$ & $c$ & $d$ & $e$ & $f$ \\
\hline 0 & 0 & 0 & 0 & 0 & 0 & 0 & 0 \\
$a$ & $a$ & 0 & 0 & 0 & 0 & 0 & 0 \\
$b$ & $b$ & $b$ & 0 & 0 & $e$ & $e$ & $b$ \\
$c$ & $c$ & $a$ & $a$ & 0 & 0 & 0 & $e$ \\
$d$ & $d$ & $d$ & $d$ & $d$ & 0 & $d$ & $e$ \\
$e$ & $e$ & $d$ & $d$ & $d$ & $d$ & 0 & $e$ \\
$f$ & $f$ & $f$ & $f$ & $f$ & $f$ & $f$ & 0
\end{tabular}

In that case; $(\mathcal{X}, \circledast, 0)$ is a d-algebra on $\mathcal{O}$ by Definition [?.], in other words, $\mathcal{X}$ is a nearness $d$-algebra. Then, $I$ is not a nearness $d$-ideal of $\mathcal{X}$, since $a, e \in \mathcal{X}, e \circledast a=d \in N_{r}(B)^{-} I$ and $a \in I$ but $e \notin I$. Hence, $I$ is an upper-near d-ideal of $\mathcal{X}$.

Example 3.9. Let $\mathcal{O}=\{0, a, b, c, d, e, f\}$ be a set of perceptual objects, $r=1, B=\left\{\varphi_{1}, \varphi_{2}, \varphi_{3}\right\} \subseteq$ $\mathcal{F}$ be a set of probe functions, and $\mathcal{X}=\{0, a, b, c, d, e\} \subset \mathcal{O}, I=\{0, a, d, e\} \subset \mathcal{X}$. Values of the probe functions

$$
\begin{aligned}
& \varphi_{1}: \mathcal{O} \rightarrow V_{1}=\left\{\beta_{1}, \beta_{2}, \beta_{3}, \beta_{4}, \beta_{5}\right\} \\
& \varphi_{2}: \mathcal{O} \rightarrow V_{2}=\left\{\beta_{1}, \beta_{2}, \beta_{3}, \beta_{4}\right\}, \\
& \varphi_{3}: \mathcal{O} \rightarrow V_{3}=\left\{\beta_{1}, \beta_{2}, \beta_{3}, \beta_{4}, \beta_{5}\right\}
\end{aligned}
$$

are given in Table 5 .

Table 5

\begin{tabular}{c|ccccccc} 
& 0 & $a$ & $b$ & $c$ & $d$ & $e$ & $f$ \\
\hline$\varphi_{1}$ & $\beta_{1}$ & $\beta_{1}$ & $\beta_{2}$ & $\beta_{2}$ & $\beta_{3}$ & $\beta_{5}$ & $\beta_{4}$ \\
$\varphi_{2}$ & $\beta_{1}$ & $\beta_{4}$ & $\beta_{2}$ & $\beta_{2}$ & $\beta_{3}$ & $\beta_{3}$ & $\beta_{2}$ \\
$\varphi_{3}$ & $\beta_{2}$ & $\beta_{1}$ & $\beta_{4}$ & $\beta_{5}$ & $\beta_{1}$ & $\beta_{3}$ & $\beta_{4}$
\end{tabular}

Now, we determine the near equivalence classes according to the indiscernibility relation of $\sim_{B_{r}}$ of elements of $\mathcal{O}:$

$$
\begin{aligned}
& {[0]_{\varphi_{1}}=\left\{y \in \mathcal{O} \mid \varphi_{1}(y)=\varphi_{1}(0)=\beta_{1}\right\}=\{0, a\}=[a]_{\varphi_{1}},} \\
& {[b]_{\varphi_{1}}=\left\{y \in \mathcal{O} \mid \varphi_{1}(y)=\varphi_{1}(b)=\beta_{2}\right\}=\{b, c\}=[c]_{\varphi_{1}},} \\
& {[d]_{\varphi_{1}}=\left\{y \in \mathcal{O} \mid \varphi_{1}(y)=\varphi_{1}(d)=\beta_{3}\right\}=\{d\},} \\
& {[e]_{\varphi_{1}}=\left\{y \in \mathcal{O} \mid \varphi_{1}(y)=\varphi_{1}(e)=\beta_{5}\right\}=\{e\},} \\
& {[f]_{\varphi_{1}}=\left\{y \in \mathcal{O} \mid \varphi_{1}(y)=\varphi_{1}(f)=\beta_{4}\right\}=\{f\} .}
\end{aligned}
$$

Then, we get that $\xi_{\varphi_{1}}=\left\{[0]_{\varphi_{1}},[b]_{\varphi_{1}},[d]_{\varphi_{1}},[e]_{\varphi_{1}},[f]_{\varphi_{1}}\right\}$. 


$$
\begin{aligned}
& {[0]_{\varphi_{2}}=\left\{y \in \mathcal{O} \mid \varphi_{2}(y)=\varphi_{2}(0)=\beta_{1}\right\}=\{0\}} \\
& {[a]_{\varphi_{2}}=\left\{y \in \mathcal{O} \mid \varphi_{2}(y)=\varphi_{2}(a)=\beta_{4}\right\}=\{a\}} \\
& {[b]_{\varphi_{2}}=\left\{y \in \mathcal{O} \mid \varphi_{2}(y)=\varphi_{2}(c)=\beta_{2}\right\}=\{b, c, f\}=[c]_{\varphi_{2}}=[f]_{\varphi_{2}}} \\
& {[d]_{\varphi_{2}}=\left\{y \in \mathcal{O} \mid \varphi_{2}(y)=\varphi_{2}(d)=\beta_{3}\right\}=\{d, e\}=[e]_{\varphi_{2}} .}
\end{aligned}
$$

Thus, we have that $\xi_{\varphi_{2}}=\left\{[0]_{\varphi_{2}},[a]_{\varphi_{2}},[b]_{\varphi_{2}},[d]_{\varphi_{2}}\right\}$.

$$
\begin{aligned}
& {[0]_{\varphi_{3}}=\left\{y \in \mathcal{O} \mid \varphi_{3}(y)=\varphi_{3}(0)=\beta_{2}\right\}=\{0\},} \\
& {[a]_{\varphi_{3}}=\left\{y \in \mathcal{O} \mid \varphi_{3}(y)=\varphi_{3}(a)=\beta_{1}\right\}=\{a, d\}=[d]_{\varphi_{3}},} \\
& {[b]_{\varphi_{3}}=\left\{y \in \mathcal{O} \mid \varphi_{3}(y)=\varphi_{3}(b)=\beta_{4}\right\}=\{b, f\}=[f]_{\varphi_{3}},} \\
& {[c]_{\varphi_{3}}=\left\{y \in \mathcal{O} \mid \varphi_{3}(y)=\varphi_{3}(c)=\beta_{5}\right\}=\{c\},} \\
& {[e]_{\varphi_{3}}=\left\{y \in \mathcal{O} \mid \varphi_{3}(y)=\varphi_{3}(f)=\beta_{3}\right\}=\{e\} .}
\end{aligned}
$$

From hence, we obtain that $\xi_{\varphi_{3}}=\left\{[0]_{\varphi_{3}},[a]_{\varphi_{3}},[b]_{\varphi_{3}},[c]_{\varphi_{3}},[e]_{\varphi_{3}}\right\}$. Therefore, for $r=1$, a set of partitions of $\mathcal{O}$ is $N_{1}(B)=\left\{\xi_{\varphi_{1}}, \xi_{\varphi_{2}}, \xi_{\varphi_{3}}\right\}$. Then, we can write

$$
N_{1}(B)^{-} \mathcal{X}=\bigcup_{\left.[x]_{\varphi_{i}} \cap \mathcal{X} \neq \emptyset\right]_{\varphi_{i}}}=\{0, a, b, c, d, e, f\}
$$

Furthermore,

$$
N_{1}(B)^{-} I=\bigcup_{[x]_{\varphi_{i}} \cap I \neq \emptyset}[x]_{\varphi_{i}}=\{0, a, d, e\} .
$$

Considering the following table of operation:

Table 6

\begin{tabular}{c|lllllll}
$\circledast$ & 0 & $a$ & $b$ & $c$ & $d$ & $e$ & $f$ \\
\hline 0 & 0 & 0 & 0 & 0 & 0 & 0 & 0 \\
$a$ & $a$ & 0 & 0 & 0 & 0 & 0 & 0 \\
$b$ & $b$ & $b$ & 0 & 0 & $b$ & $b$ & 0 \\
$c$ & $c$ & $c$ & $c$ & 0 & $c$ & $c$ & 0 \\
$d$ & $d$ & $d$ & $d$ & $d$ & 0 & $d$ & 0 \\
$e$ & $e$ & $e$ & $e$ & $e$ & $e$ & 0 & 0 \\
$f$ & $f$ & $f$ & $f$ & $f$ & $f$ & $f$ & 0
\end{tabular}

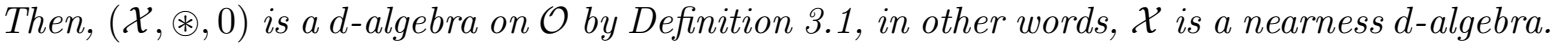
Moreover, $I$ is a nearness d-ideal of $\mathcal{X}$.

Let $\mathcal{X}$ be a nearness $d$-algebra. Let $X \circledast Y=\{x \circledast y \mid x \in X$, and $y \in Y\}$, where $X$ and $Y$ are subsets of $\mathcal{X}$.

Lemma 3.10. Let $\mathcal{X}$ be a nearness d-algebra. If $\sim_{B_{r}}$ is a congruence indiscernibility relation on $\mathcal{X}$, then $[x]_{B_{r}} \circledast[y]_{B_{r}} \subseteq[x \circledast y]_{B_{r}}$ for all $x, y \in \mathcal{X}$. 
Definition 3.11. Let $\mathcal{X} \subseteq \mathcal{O}$ and $\left(\mathcal{O}, \mathcal{F}, \sim_{B_{r}}, N_{r}(B)\right)$ be a weak near approximation spaces, $B_{r} \subseteq$ $\mathcal{F}$ where $r \leq|B|$ and $B \subseteq \mathcal{F}, \sim_{B_{r}}$ be an indiscernibility relation on $\mathcal{O}$. Then, $\sim_{B_{r}}$ is called a complete congruence indiscernibility relation on nearness d-algebra $\mathcal{X}$, if $[a]_{B_{r}} \circledast[b]_{B_{r}}=[a \circledast b]_{B_{r}}$ for all $a, b \in \mathcal{X}$.

Theorem 3.12. Let $\mathcal{X}$ be a nearness d-algebra, and I be a non-empty subset of $\mathcal{X}$. If $\sim_{B_{r}}$ is a complete congruence indiscernibility relation on $\mathcal{X}$, and $I$ is nearness $d$-ideal of $\mathcal{X}$, then $I$ is an upper-near d-ideal of $\mathcal{X}$.

Proof. Let $a, b \in \mathcal{X}$ such that $a \circledast b, b \in N_{r}(B)^{-} I$. Then, $[a \circledast b]_{B_{r}} \cap I \neq \emptyset$ and since $\sim_{B_{r}}$ is a complete congruence indiscernibility relation, we have $[a]_{B_{r}} \circledast[b]_{B_{r}} \cap I \neq \emptyset$ and $[b]_{B_{r}} \cap I \neq \emptyset$. Therefore, there exist $c, d \in I$ such that $c \in[a]_{B_{r}} *[b]_{B_{r}} \cap I \neq \emptyset$ and $d \in[b]_{B_{r}} \cap I \neq \emptyset$. Thus, we have $c=x \circledast y ; x \in[a]_{B_{r}}, y \in[b]_{B_{r}}$, and so we get $a \sim_{B_{r}} x$ and $b \sim_{B_{r}} y$, and also $b \sim_{B_{r}} d$. Hence, we obtain $y \sim_{B_{r}} d$ and so $[y]_{B_{r}}=[d]_{B_{r}}$. Since $d \in I$, we have $y \in I$. Combining $c=x \circledast y \in I$ and using $y \in I$, we have $x \in I$ by hypothesis. In this case, $[a]_{B_{r}} \cap I \neq \emptyset$ implies $a \in N_{r}(B)^{-} I$. Similarly, (UNI3) is obtained. On the other hand, we have $a \circledast a=0 \in N_{r}(B)^{-} I$ by hypothesis. This comletes the proof.

\section{Conclusions}

As a recent study of nearness $B C K$-algebras, this paper shows some notion of nearness $d$-algebras which is a generalization of nearness $B C K$-algebras. Also, it is defined that the notions of nearness $d$-subalgebra, nearness $d$-ideal in nearness $d$-algebras. Afterward, we investigate relations among them and give some examples. We believe that these properties will be more useful theoretical development for $d$-algebras theory. One can consider others types of algebra like nearness $B C C$ algebra, nearness $B C H$-algebra, nearness $P U$-algebra, nearness subtraction algebra and etc. based on the ideas and results of this paper.

\section{$5 \quad$ Acknowledgement}

The author(s) would like to thank the anonymous reviewers for their careful reading of this paper and for their helpful comments.

\section{References}

[1] P.J. Allen, H.S. Kim, J. Neggers, Companion d-algebras, Mathematica Slovaca, 57 (2007), 93-106.

[2] E. İnan, M.A. Öztürk, Near groups on nearness approximation spaces, Hacettepe Journal of Mathematics and Statistics, 41(4) (2012), 545-558.

[3] E. İnan, M.A. Öztürk, Erratum and notes for near groups on nearness approximation spaces, Hacettepe Journal of Mathematics and Statistics, 43(2) (2014), 279-281.

[4] E. İnan, M.A. Öztürk, Near semigroups on nearness approximation spaces, Annals of Fuzzy Mathematics and Informatics, 10(2) (2015), 287-297.

[5] Y.B. Jun, Roughness of ideals BCK-algebras, Scientiae Mathematicae Japonicae, 7 (2002), $115-119$. 
[6] J. Meng, Y.B. Jun, BCK-algebras, Kyung Moon SA Co, Seoul-Korea, 1994.

[7] D. Molodtsov, Soft set theory - first results, Computers and Mathematics with Applications, 37 (1999), 19-31.

[8] J. Neggers, Y.B. Jun, H.S. Kim, On d-ideals in d-algebras, Mathematica Slovaca, 49 (1999), $243-251$.

[9] J. Neggers, H.S. Kim, On d-algebras, Mathematica Slovaca, 49 (1999), 19-26.

[10] M.A. Öztürk, Semirings on weak nearness approximation spaces, Annals of Fuzzy Mathematics and Informatics, 15(3) (2018), 227-241.

[11] M.A. Öztürk, Prime ideals of gamma semigroups on weak nearness approximation spaces, Asian-European Journal of Mathematics, 12(5) (2019), 1950080 (10 pages).

[12] M.A. Öztürk, İ. Çelik Siner, Y.B. Jun, Nearness BCK-algebras, International Journal of Open Problems in Computer Science and Mathematics, 8(4) (2015), 37-57.

[13] M.A. Öztürk, E. İnan, Soft nearness approximation spaces, Fundamenta Informaticae, 124(1) (2013), 231-250.

[14] M.A. Öztürk, Y.B. Jun, Nobusawa gamma nearness rings, New Mathematics and Natural Computation, 15(2) (2019), 373-394.

[15] M.A. Öztürk, Y.B. Jun, Quotient nearness BCK-algebras, (Submitted).

[16] M.A. Öztürk, Y.B. Jun, A. İz, Gamma semigroups on weak nearness approximation spaces, Journal of International Mathematical Virtual Institute, 9 (2019), 53-72.

[17] M.A. Öztürk, İ. Temur, Prime ideals of nearness semirings, Communications Faculty of Sciences University of Ankara Series A1 Mathematics and Statistics, 68(2) (2019), 1867-1878.

[18] M.A. Öztürk, M. Uçkun, E. İnan, Near groups of weak cosets on nearness approximation spaces, Fundamenta Informaticae, 133 (2014), 433-448.

[19] M.A. Öztürk, H. Yazarli, Nobusawa gamma nearness semirings, New Mathematics and Natural Computation, (In press).

[20] Z. Pawlak, Rough sets, International Journal of Computer and Information Sciences, 11(5) (1982), 341-356.

[21] J.F. Peters, Near sets: General theory about nearness of objects, Applied Mathematical Sciences (Ruse), 1(53-56) (2007), 2609-2629.

[22] J.F. Peters, Near sets: Special theory about nearness of objects, Fundamenta Informaticae, 75 (2007), 407-433.

[23] J.F. Peters, Classification of perceptual objects by means of features, International Journal of Information Technology and Intelligent Computing, 3(2) (2008), 1-35.

[24] J.F. Peters, Near sets: An introduction, Mathematics in Computer Science, 7(1) (2013), 3-9.

[25] J.F. Peters, S.A. Naimpally, Applications of near sets, Notices of the American Mathematical Society, 59(4) (2012), 536-542. 
[26] J.F. Peters, P. Wasilewski, Foundations of near sets, Information Sciences, 179(18) (2009), 3091-3109.

[27] L.A. Zadeh, From circuit theory to system theory, Proceedings of the Institution of Radio and Electronics Engineers, 50(5) (1962), 856-865.

[28] L.A. Zadeh, Fuzzy sets, Information and Control, 8 (1965), 338-353. 